\title{
Effect of attachment-based interventions on prenatal attachment: a protocol for systematic review
}

\author{
Kobra Salehi ${ }^{1}$, Fariba Taleghani ${ }^{2}$ and Shahnaz Kohan ${ }^{2^{*}}$ (D)
}

\begin{abstract}
Background: Parental attachment was defined as: series of inner behaviors that would cause the infant to develop an intimate relation with his/her parents. This emotional relationship is formed long before birth during the pregnancy and has been associated with psychosocial outcomes for women and children. This relationship is known as one of the major components of the child's social and emotional development. Parents' relationship with their fetus could be strengthened using various strategies, but efforts to augment the maternal-fetal relationship have not always been successful. This study aims to conduct a comprehensive systematic review and a metaanalysis survey of the effects of attachment-based interventions on prenatal attachment.

Methods: A comprehensive search of relevant randomized and quasi-randomized controlled trials will be performed in EMBASE (via Scopus), ProQuest, Pubmed, Scopus, Ovid and Web of Science, The Cochrane Central Register of Controlled Trials, SID, Maglran, Irandoc, Barakat Knowledge Network System and Iranian registry of clinical trials website as Iranian databases using English and Persian keywords such as prenatal attachment, relationship, maternal attachment. Only randomized controlled clinical trials conducted between 2000 and 2016 will be included in this review. The study will be selected if their participants were expectant mothers, their partners or both. Our primary outcome will be the effect size of intervention. The quality of experimental studies will be evaluated using CONSORT checklist and Study Quality Guide by Cochrane Consumers and Communication Review Group.

Two authors will independently assess the eligibility of the studies. Any disagreements will be resolved through a third reviewer. The risk of bias will be independently determined using the Cochrane Risk of Bias Tool. The quality of the papers will be assessed based on the CONSORT checklist. If possible quantitative data will be pooled in statistical meta-analyzing with random effect model.

Discussion: In this review the current state of knowledge on prenatal attachment is examined. Effectiveness of attachment-based interventions during pregnancy is analyzed. Finally, practice and research implications based on analysis of the current status of maternal-fetal attachment are identified. The expected findings will help healthcare providers to develop pregnant women and infants' health when offering prenatal care.
\end{abstract}

Keywords: Attachment, Maternal-fetal relationship, Review, Paternal fetal relationship

\footnotetext{
* Correspondence: kohan@nm.mui.ac.ir

${ }^{2}$ Nursing and Midwifery Care Research Center, Faculty of Nursing and

Midwifery, Isfahan University of Medical Sciences, Isfahan, Iran

Full list of author information is available at the end of the article
}

(c) The Author(s). 2019 Open Access This article is distributed under the terms of the Creative Commons Attribution 4.0 International License (http://creativecommons.org/licenses/by/4.0/), which permits unrestricted use, distribution, and reproduction in any medium, provided you give appropriate credit to the original author(s) and the source, provide a link to the Creative Commons license, and indicate if changes were made. The Creative Commons Public Domain Dedication waiver (http://creativecommons.org/publicdomain/zero/1.0/) applies to the data made available in this article, unless otherwise stated. 


\section{Plain English summary}

Attachment is a close and intimate relationship with people like mother, father, sister, brother, partner, child and close friends. Parental attachment was defined as: series of inner behaviors that would cause the infant to develop an intimate relation with his/her parents. This emotional relationship is formed long before birth during the pregnancy. Maternal-fetal relationship has been associated with psychosocial outcomes for women and children. This relationship is known as one of the major components of the child's development. Father's attachment to the fetus plays an important role in accepting the paternal identity, desirable pregnancy outcomes and improvement of maternal-fetal health; The pattern of prenatal attachment during pregnancy could be changed and parents' preparation for developing a joyful relationship with their fetus could be strengthened using various strategies. Thus some researchers have considered the prenatal period to be a proper opportunity to build a desirable mother-infant bond before birth, but efforts to augment the maternal-fetal relationship have not always been successful and the effect of the proposed interventions in this field are not yet determined. Also based on our knowledge and understanding, no systematic review has evaluated the effect of attachment-based interventions on prenatal attachment. The aim of this study is to conduct a comprehensive review of the effects of attachment-based interventions on prenatal attachment. Therefore, the results of this study can potentially help to select appropriate interventions for prenatal attachment.

\section{Background}

Attachment is a close and intimate relationship with people like mother, father, sister, brother, partner, child and close friends [1]. Parental attachment was defined as: series of inner behaviors that would cause the infant to develop an intimate relation with his/her main caregiver [2]. This emotional relationship is formed long before birth during the pregnancy [3-7].

Maternal-fetal relationships have been associated with psychosocial outcomes for women and children [8]. This relationship is known as one of the major components of the child's social and emotional development $[5,6,9]$; in this relationship, the child would learn the manner of relating to and communicating with others and therefore, it is considered as the foundation for formation of child's future behaviors [10]. As well, attachment to one's fetus may contribute to lower risk of child abuse [11]. Understanding MFR is necessary for understanding adaptation in pregnancy [8].

The maternal-fetal attachment is closely related to other important processes such as motherhood and maternal identity $[12,13]$. Maternal fetal attachment is a unique opportunity for mother and child to form a unique relationship of importance and value [14].

Father's attachment to the fetus plays an important role in accepting the paternal identity, desirable pregnancy outcomes and improvement of maternal-fetal health; fathers with higher attachment to their fetus are usually more sensitive toward healthy behaviors of their wives for receiving prenatal care [15]. Ideally, additional knowledge about MFA could develop the interventions that begin before birth and prevent poor mother-child attachment from being an "inevitable sequence of events." [16]. the pattern of prenatal attachment during pregnancy could be changed [5] and parents' preparation for developing a joyful relationship with their fetus could be strengthened using various strategies. Thus some researchers have considered the prenatal period to be a proper opportunity to build a desirable mother-infant bond before birth [17].

Some of the measures in this regard include intervention using fetal palpation [18], attention to the movements of the fetus $[1,19]$, and having singing, dancing, and massage-through-the-womb sessions [20], guide imagery [21], but the effect of the proposed interventions in his field are not yet determined [19, 22, 23]. But efforts to augment the maternal-fetal relationship have not always been successful [8]. During the recent years, by clarifying the necessity of evidence-based practice in clinical majors including midwifery, at first the studies that have been conducted in the field of attachment, their results and the necessary studies for future must be determined. When the goal is to make a recommendation regarding the effectiveness of an intervention, systematic review is necessary [24]. Review studies which are necessary for guiding the policies and making the decisions, would be helpful in planning for performing new researches. In addition, meta-analysis can also resolve controversy where there have been conflicting results [25]. Results of the present study could help the providers of prenatal care in making more appropriate evidence-based decisions in the field of prenatal attachment.

In this integrative review the current state of knowledge on prenatal attachment is examined. Effectiveness of attachment-based interventions during pregnancy is analyzed. Finally, practice and research implications based on analysis of the current status of maternal-fetal attachment are identified.

\section{Objectives}

This Systematic review will aim to clarify what prenatal interventions have been performed for improvement of prenatal attachment? Whether interventions are effective in promoting paternal attachment? Does this improvement depend on time and duration of intervention? 


\section{Methods}

The guidelines of PRISMA-P (Preferred Reporting Items for Systematic Review and Meta-Analysis Protocols) were followed while reporting the study protocol [26].

\section{Data sources}

This study will be a systematic review about the effectiveness of prenatal interventions on parental attachment. Databases including EMBASE (via Scopus), ProQuest, Pubmed, Scopus, Ovid and Web of Science, The Cochrane Central Register of Controlled Trials, SID, MagIran, Irandoc, Barakat Knowledge Network System and Iranian registry of clinical trials website as Iranian databases will be systematically searched.

The search of ongoing clinical trials will be performed in following databases:

http://www.isrctn.com/

www.clinicaltrials.gov

http://apps.who.int/trialsearch/

\section{Types of studies}

Randomized and quasi-randomized controlled trials published between 2000 and 2016 included cluster and cross over, blinded and non-blinded design will be included in this review. Observational and qualitative studies, along with letters to the editor, case series, and case reports will be excluded. No language limitations will be imposed during the search. In the cases that the language used in an article is other than Persian or English, we will ask for a translator to translate the article.

\section{Type of participants}

The studies will be selected if their participants were expectant mothers or their partners or both.

\section{Types of intervention/ comparisons}

This review will include studies that assessed the effects of any kind of interventions during pregnancy on parental attachment, compared the intervention with control group or along with other interventions. No limit will be imposed on intervention type and time.

\section{Outcome}

The studies will be selected if they provided objective measurement of prenatal attachment before and after their intervention. Our primary outcome will be the effect size of intervention. The rate of changes in attachment score in the study groups will be compared. Outcome measurement will be based on Cranley's Maternal Fetal Attachment Scale [27] or Muller Prenatal Attachment Inventory [7] or Maternal Antenatal Attachment Scale by Condon [28].
Search strategies for identification of studies

A comprehensive list of keywords and medical subject heading $(\mathrm{MeSH})$ terms will be provided for each part of the PICOC. For example for the population, phrases such as pregnant woman, expectant mother/father, husband/partner of pregnant women and expectant parent will be used. Different combinations of the keywords which will be combined using Boolean operators AND and OR. Further relevant keywords and Boolean operators will also be selected for a change of strategy in each particular database. (Table 1).

\section{Searching other resources}

Also, the archives of all the Persian-language journals in the fields of midwifery, reproductive health, family and psychology, key journals, Government reports, theses and dissertations, papers published by research committees, and abstracts of papers presented at different conferences and seminars, will be manually searched based on the keywords.

\section{Selection of relevant studies}

After completing the search in each section of PICOC, studies will be sorted based on their publication year and the titles of the studies will be reviewed to find repetitions. Then selected articles will be determined. The abstracts of the articles will be studied and the inclusion criteria will be evaluated. To avoid the selection bias, decisions about including or excluding the studies will be made based on previously determined inclusion and exclusion criteria. These criteria will be developed based on the objectives of the review and its components. Published original articles between 2000 to 2016 in any languages that have been done among study population including pregnant women, their husbands or both while having a clear indication of measurement attachment during pregnancy and evaluated the effects of interventions on prenatal attachment, will be selected in order to assess their full texts.

Table 1 Example for Keywords combinations

Maternal/Paternal And Attachment AND/OR Fetus
Prenatal/Pregnancy AND Attachment
Parental/Expectant Mother/Father AND Attachment
Paternal/Maternal AND Attachment
Maternal/Paternal AND Fetal AND Relation
Prenatal/Pregnancy AND Relationship
Mother/father AND Fetal AND Relation
Mother/Father AND Fetal AND Attachment
Similar phrases and Persian synonyms were also will be used.




\section{Data extraction}

Data will be collected as follows:

1. Research information (the first author, geographic location of study setting, year of publication, beginning and end dates, research design, sample size, duration of intervention).

2. Characteristics of the participants (age, gender, inclusion and exclusion criteria, score of attachment, measurement tools).

3. Intervention and comparisons of the details (number of groups, conducting intervention, type of intervention, sample loss).

4. Outcome measures (explanations about administered measurement tools and methods of evaluating outcomes).

Extracted data from each study will be reviewed and organized into the form of tables.

\section{Risk of bias within the studies}

To increase the reliability of the study's results and to prevent bias in data entrance, searching, study selection, making decision for inclusion or exclusion of the studies and evaluating the full texts will be conducted by two independent reviewers. The process will be monitored by the supervisor with any disagreements resolved by discussion to reach consensus.

\section{Quality assessment of studies}

In the step of data management and processing, for assessment of the studies' quality, a standard assessment appropriate for the type of the study will be used. The quality of experimental studies will be evaluated using CONSORT checklist [29] and Study Quality Guide by Cochrane Consumers and Communication Review Group [30]. Only studies that scored over 15 on the CONSORT checklist will be included.

\section{Data synthesis}

If possible quantitative data will be pooled in statistical meta-analyzing using Review Manager (Rev Man) software. Meta-analysis is undertaken in two stages: the first beings analysis of the outcome and summary statistics effect measure. The second stage of a meta-analysis is the statistics from each individual study are pooled to give an overall estimate. Random effect model of meta-analysis will conduct for outcomes. This model assumes that studies are taken from different effect size and considering the extent of variation and heterogeneity. This can be due to factors such as study populations, the manner in which the intervention was implemented or even the reliability of the methodology for measuring the effect. All results will be subject to double data entry. Effect sizes expressed as weight mean differences (for continuous data) and OR (for categorical data) with 95\% confidence interval. Results from meta-analysis are presented using forest plot. If pooling data is not possible, the findings will be presented in narrative review using thematic summaries and tables.

\section{Discussion}

This protocol will be the updated review of the available literature about the effects of prenatal intervention on the paternal attachment. Intervention studies can provide the strongest evidence that is the strength of this review.

Considering the uncertain effects of attachment-based interventions on prenatal attachment, it was decided to perform this study. This is the first systematic review that will perform without any language restrictions; this study will draw a comprehensive image of the attachment-based interventions for prenatal attachment. By analyzing the existing evidences, it is possible to design appropriate interventions and it is expected that the results of this study will have the same effect. It also identifies existing deficiencies for further research. Information of previous studies may also help in future research.

Due to increase attention to the early development of children, prenatal period can be an appropriate onset to improve outcomes. If the efficacy of prenatal interventions will demonstrate in the present study, given to the importance of attachment, these strategies could be useful for women and children. Also women unsure of their attachment may respond to appropriate interventions, and women unaware of or unconcerned about their attachment to their fetus may benefit from effective interventions.

\section{Strengths and limitations of this study}

Systematic reviews will provide the reliable evidence for informed decisions. To the best of our knowledge, no meta-analysis has been conducted on this topic. One limitation of this study is that the authors are only fluent in Persian and English. Therefore a translator will be required when the papers are published in other languages.

\section{Abbreviations}

CONSORT: Consolidated Standards of Reporting Trials; PICOC: Population, Interventions, Comparisons, and the Outcomes related to the objectives and Context of the study

\section{Acknowledgments}

This paper is a part of reproductive health PhD thesis (N. 395362). The authors would like to thank Isfahan University of Medical Sciences for supporting this research.

\section{Funding}

This research protocol was supported by the Isfahan University of Medical Sciences, Isfahan, Iran (research no. 395362). 


\section{Availability of data and materials}

Not applicable.

\section{Authors' contributions}

KS, FT and SK, were involved in study conception, design and drafting of the manuscript. KS wrote the first draft of this study protocol. SK, FT was responsible for assessment of eligibility criteria of papers. KS was responsible for evaluation of full text and data gathering. SK and FT reviewed the first draft of the protocol and manuscript. SK was responsible for coordinating the study. KS will be responsible for interview with participants, description and data analysis. All authors will review and will involve in data analysis. All authors read and approved the final manuscript.

\section{Ethics, consent and permission}

This study will review the findings of previous researches.

\section{Ethics approval}

The protocol of this study was registered at Isfahan University of Medical Sciences by research No 395362 and Ethical approval for this study has been obtained by the ethics committee affiliated with Isfahan University of Medical Sciences, Isfahan, Iran (reference number: IR.MUI.REC.1395.3.362).

\section{Consent for publication}

Not applicable.

\section{Competing interests}

The authors declare that they have no competing interests.

\section{Publisher's Note}

Springer Nature remains neutral with regard to jurisdictional claims in published maps and institutional affiliations.

\section{Author details}

${ }^{1}$ Student Research Committee, School of Nursing and Midwifery, Isfahan University of Medical Sciences, Isfahan, Iran. ${ }^{2}$ Nursing and Midwifery Care Research Center, Faculty of Nursing and Midwifery, Isfahan University of Medical Sciences, Isfahan, Iran.

\section{Received: 15 March 2019 Accepted: 25 March 2019}

\section{Published online: 05 April 2019}

\section{References}

1. Salehi K, Salehi Z, Shaali M. The effect of education of fetal movement counting on maternal-fetal attachment in the pregnant women: a randomized controlled clinical trial. Int J Pediatr. 2017;5(4):4699-06.

2. Bowlby J. Attachment and loss, vol. 1. New York: Basic Books; 1969.

3. Akbarzadeh M, Moattari M, Abootalebi M. Effect of the BASNEF model on maternal-fetal attachment in the pregnant women referring to the prenatal clinics affiliated to Shiraz University of Medical Sciences. Iranian Journal of Neonatology. 2017:8(3). https://doi.org/10.22038/ijn.2017.20521.1229.

4. Ossa X, Bustos L, Fernandes L. Prenatal attachment and associated factors during the third trimester of pregnancy in Tumeco, Chile. Midwifery. 2012; 28:689-96.

5. Gurol A. \& Palot S. The effects of baby massage on attachment between mother and their infants. Asian Nursing Research. 2012:6:35-41.

6. Sedgmen B, Mcmahon G, Carins D, Benzie R, Woodfied R. The impact of two-dimensional versus three dimensional ultrasound exposure on maternal-fetal attachment and maternal health behavior in pregnancy Ultrasound obstetric gynecology. 2006:27:245-51.

7. Muller ME. Development of the prenatal attachment inventory. West J Nurs Res. 1993;15(2):199-215.

8. Walsh J, Hepper EG, Marshall BJ. Investigating attachment, caregiving, and mental health: a model of maternal-fetal relationships. BMC Pregnancy and Child birth. 2014;14:383.

9. Ruschel P, Zielinsky P, Grings C, Pimentel J, Azevedo L, Paniagua R, et al. Maternal fetal attachment and prenatal diagnosis of heart disease. European Journal of obstetrics and gynecology and reproductive biology. 2014;174:70-5.

10. Sroufe L, Egeland B, Carlson E, Collins W. The development of the person: the Minnesota study of risk and adaptation from birth to adulthood. New York: Guilford Press; 2005
11. Zimerman A, Doan H. Prenatal attachment and other feeling and thoughts during pregnancy in three groups of pregnant women. Journal of prenatal and perinatal psychology and health. 2003;18:2.

12. Meleis A. Transition theory: middle range and situation specific theories in nursing research and Practicies. New York: Springer Publishing Company; 2010

13. Mercer R. Becoming a mother versus maternal role attainment. J Nurs Scholarsh. 2004;36:226-32.

14. Abasi E, Tahmasebi H, Zafari M. Nasiri Takami N. Assessment the effective factors of maternal fetal attachment in pregnant women. Life Science Journal. 2012;9(1):68-75.

15. Salehi K, Sh K, Taleghani F. Factors and interventions associated with parental attachment during pregnancy in Iran: a systematic review. Int J Pediatr. 2018:6(1):6823-42

16. Brandon A, Pitts S, Denton W, Stringer A, Evans H. A history of the theory of prenatal attachment. Prenat prinat psychol health. 2009:23:201-22.

17. Akbarzade M, Rafiee B, Asadi N, Nematollahi A, Taheri M. Comparison of the effects of attachment and relaxation instruction on the third trimester depression and post-partum blues. NJOG. 2017;23(1):17-21.

18. Bryan A. Enhancing parent-child interaction with a prenatal couple intervention. MCN. 2000:25(3):139-44.

19. Saastad E, Israel P, Gunnes N, Froen F. Fetal movement counting - effects in maternal fetal attachment: a multicentre randomized controlled trial. Birth. 2011;38(4):282-93

20. Bellieni C, Ceccarelli D, Rossi F, Buonocore G, Maffei M, Perrone S, et al. Is prenatal bonding enhanced by prenatal education courses? Minerva Ginecol. 2007:59(2):125-9.

21. Kordi M, Farsanghari $\mathrm{M}$, Esmaily $\mathrm{H}$. Effect of guide imagery on maternal fetal attachment in nulipaou women with unplanned pregnancy. JMRH. 2016; 4(4):723-31

22. Kim J, Cho K. The effect of mother-fetus interaction promotion program of talking and tactile stimulation on maternal-fetal attachment. Korean J Child Health Nurs. 2004;10(2):153-64.

23. Chang S, Kim K, Kim E. Change of maternal- fetal attachment and selfefficacy for delivery after the taekyo-prespective prenatal class. Korean Journal of Women Health Nurs. 2001;7(1):7-17.

24. Brown SJ. Evidence-based nursing : the research practice connection: Jones and Bartlett Publishers; 2009

25. Coughlan M, Cronin P, Ryan F. Doing a literature review in nursing and social care: SAGE; 2013.

26. PRISMA Checklist. available online at: http://www.prisma-statement.org/ statement.htm. Accessed 30 Feb 2018.

27. Cranley MS. Development of a tool for the measurement of maternal attachment during pregnancy. Nurs Res. 1981;30:281-284.

28. Condon JT. The assessment of antenatal emotional attachment: development of a questionnaire instrument. Br J Med Psychol. 1993;66:167-83.

29. CONSORT 2010 Checklist of information to include when reporting a randomized trial. http://uww.consort-statement.org/consort-statement/ checklist.

30. Wells GA, Shea B, O'Connel D, Welch V, Losos M, Tugwell P. The NewcastleOttawa scale (NOS) for assessing the quality of non-randomized studies in meta-analyses. http://www.ohri.ca/programs/clinical_epidemiology/oxford.asp. Accessed 30 Feb 2018

Ready to submit your research? Choose BMC and benefit from:

- fast, convenient online submission

- thorough peer review by experienced researchers in your field

- rapid publication on acceptance

- support for research data, including large and complex data types

- gold Open Access which fosters wider collaboration and increased citations

- maximum visibility for your research: over $100 \mathrm{M}$ website views per year

At BMC, research is always in progress.

Learn more biomedcentral.com/submissions 